Research Paper

\title{
Expression of NDRG2 in Human Colorectal Cancer and its Association with Prognosis
}

\author{
Wenjing Chen ${ }^{1 *}$, Jianhong Peng ${ }^{2 *}$, Qingjian $\mathrm{Ou}^{2,3^{*}}$, Yongshan $\mathrm{Wen}^{2}$, Wu Jiang ${ }^{2}$, Yuxiang Deng ${ }^{2}$, Yujie Zhao ${ }^{2}$, \\ Desen Wan ${ }^{2}$, Zhizhong Pan ${ }^{2}$, Yujing Fang ${ }^{2,3}$ \\ 1. Department of Clinical Laboratory, the First Affiliated Hospital, Jinan University, Guangzhou 510630, China. \\ 2. Department of Colorectal Surgery, Sun Yat-sen University Cancer Center; State Key Laboratory of Oncology in South China; Collaborative Innovation \\ Center for Cancer Medicine, Guangzhou, Guangdong 510060, P. R. China. \\ 3. Department of Experimental Research, Sun Yat-sen University Cancer Center; State Key Laboratory of Oncology in South China; Collaborative Innovation \\ Center for Cancer Medicine, Guangzhou, Guangdong 510060, P. R. China. \\ *Wenjing Chen, Jianhong Peng and Qingjian Ou contributed equally to this work. \\ $\bowtie$ Corresponding authors: Yujing Fang and Zhizhong Pan, Tel.: +86-87342280; Fax: +86-20-87343637; E-mail: fangyj@sysucc.org.cn
}

(c) Ivyspring International Publisher. This is an open access article distributed under the terms of the Creative Commons Attribution (CC BY-NC) license (https://creativecommons.org/licenses/by-nc/4.0/). See http://ivyspring.com/terms for full terms and conditions.

Received: 2018.11.10; Accepted: 2019.04.25; Published: 2019.06.09

\begin{abstract}
Objective: As a member of the N-myc downregulated gene family, N-Myc downstream-regulated gene 2 (NDRG2) contributes to tumorigenesis of various types of cancer. The expression status of NDRG2 in colorectal cancer (CRC) and its prognostic value remain to be elucidated. The goal of this study was to determine the expression pattern of NDRG2 in human CRC and its association of NDRG2 expression with prognosis.

Methods: Immunohistochemistry was used to determine the level of NDRG2 expressions in 316 CRC tissues. The medical records of consecutive CRC patients undergoing primary tumor resection from September 2000 to February 2015 were retrospectively selected. Then, we compared to specific clinicopathological features in patients with different level of NDRG2 expressions. The correlation of NDRG2 expression with 3-year survival rate was assessed by Kaplan-Meier method and Cox regression modeling.

Results: NDRG2 was expressed in 94.6\% (299/316) of CRC tissues. The median IHC score of NDRG2 expression was significantly lower in tumor tissues compared with that of tumor-adjacent normal tissues [4.50(range $0.00-12.00$ ) vs. 10.00 (range $0.00-12.00$ ), $P<0.001$ ].Survival analysis indicated that patients with low NDRG2 expression had poorer 3-year OS than those with high NDRG2 expression $(59.9 \%$ vs. $76.6 \%, P=$ $0.017)$. Low NDRG2 expression also presented a significantly poorer 3-year OS rate in patient with stage IV disease $(29.4 \%$ vs. $56.5 \%, P=0.002)$, liver metastasis $(32.2 \%$ vs. $54.7 \%, P=0.005)$ and those receiving liver resection( $56.5 \%$ vs. $71.9 \%, P=0.012$ ). Multivariate analysis indicated that high NDRG2 expression was independently associated with poor OS (hazard ratio [HR]: 1.499; 95\% confidence interval [Cl]: 1.037-2.165; P $=0.031$ ).

Conclusions: Low expression of NDRG2 was associated with unfavorable prognosis in CRC patients with primary tumor resection. Detection of NDRG2 expression might be useful for providing valuable information of individualized therapy for CRC patients.
\end{abstract}

Key words: NDRG2, colorectal cancer, expression, prognosis

\section{Introduction}

Colorectal cancer (CRC) is one of the most common cancers, and it is the leading cause of cancer death in China and worldwide [1, 2]. Despite improvements in the comprehensive treatment and management of CRC patients in recent years, the 5 -year overall survival (OS) rate for advanced CRC is approximately $64.4-66.6 \%$ [3]. Surgical resection offers the possibility of a cure for CRC patients and remains the most effective treatment. Nevertheless, we previously reported that approximately $20.1 \%-57.3 \%$ of patients who first undergo complete tumor resection experience disease recurrence $[4,5]$. Recent molecular analyses of CRC identified a series of genetic biomarkers, such as RAS, BRAF, APC and 
PIK3CA, that can aid in the identification of patients at a high risk of disease recurrence after surgery [6-8]. Thus, identifying more novel markers to screen various prognostic risk subgroups and to gain a deeper understanding of the molecular mechanisms involved in CRC progression will allow for postoperative therapy development to improve the current treatment options.

$\mathrm{N}$-myc downstream-regulated gene 2 (NDRG2) is a member of the N-myc downregulated gene family, which belongs to the alpha/beta hydrolase superfamily $[9,10]$. NDRG2 is involved in cellular differentiation and human nervous system disorders $[11,12]$. Additionally, accumulative evidence indicated that NDRG2 functions as a tumor suppressor that inhibits tumor proliferation, adhesion, and invasion [13, 14]. Moreover, NDRG2 expression is decreased in tumor tissues, and its suppressed expression is associated with poor prognosis in many types of cancers, including lung cancer, esophageal squamous cancer and pancreatic cancer [15-18]. Recent studies have also indicated that NDRG2 abrogated TGF- $\beta$-induced epithelial-mesenchymal transition (EMT) and further inhibited the invasion and migration of CRC cells [19, 20]. However, the possible prognostic value of NDRG2 in CRC patients remains to be further elucidated. Understanding the clinical significance of NDRG2 in CRC may help to formulate postoperative treatment strategies for CRC patients.

In the present study, we aimed to detect NDRG2 expression levels in 316 samples from patients with stage II-IV disease and explore the relationship between NDRG2 expression levels and clinical pathological information. Furthermore, the relationship between NDRG2 expression and 3-year survival outcomes for various stages of CRC was also assessed. The results of the present study may answer the question of whether NDRG2 might serve as a novel prognostic marker for CRC.

\section{Materials and methods}

\section{Patients and collection of samples}

A total of 316 patients with primary CRC who underwent primary tumor resection from September 2000 to February 2015 at the Department of Colorectal Surgery at Sun Yat-sen University Cancer Center were recruited for the present study. All included patients met the following inclusion criteria: (1) histologically confirmed colorectal adenocarcinoma; (2) no preoperative anti-cancer therapy; (3) radical resection for colorectal primary tumor; and (4) postoperative follow-up for at least 3 months. The patient demographics, tumor characteristics, adjuvant chemothe- rapy and follow-up data were collected from the electronic medical record system. The treatment strategy and operability of liver metastases for the patients with liver metastasis were assessed by a multidisciplinary team (MDT). Paraffin-embedded samples were obtained from resected tumors and confirmed by pathological review. Normal tissues that were at least $5 \mathrm{~cm}$ away from the tumor were obtained from the patients. CRC specimens were staged in accordance with the 2010 American Joint Committee on Cancer staging system. The study was approved by the Institutional Research Ethics Committee of Sun Yat-sen University Cancer Center (Approval number: B2017-042-01), and all samples were taken with the informed consent of the donors.

\section{Immunohistochemical staining}

Immunohistochemistry was carried out as we previously described [21, 22]. First, the tissue microarrays (TMAs) were constructed from the paraffinembedded samples using a tissue array instrument (Beecher Instruments, Sun Prairie, WI, USA). Then, the TMAs were sectioned continuously into 4-mmthick sections, which were dewaxed in xylene, rehydrated and rinsed in graded ethanol solutions. The antigens were retrieved by heating the tissue sections at $100^{\circ} \mathrm{C}$ for $5 \mathrm{~min}$ in an EDTA solution $(1 \mathrm{mmol} / \mathrm{L}$, $\mathrm{pH}$ 8.0). The sections were then immersed in a $0.3 \%$ hydrogen peroxide solution for $10 \mathrm{~min}$ and rinsed with phosphate buffered saline (PBS) for $5 \mathrm{~min}$. All sections were incubated with an NDRG2 primary antibody (1:10000 dilution, ab174850; Abcam, Cambridge, UK) at $4{ }^{\circ} \mathrm{C}$ overnight. After washing with $1 \times$ PBS, the sections were treated with a goat antibody against a mouse/rabbit secondary antibody (Envision; Dako, Glostrup, Denmark) at $37.5^{\circ} \mathrm{C}$ for $30 \mathrm{~min}$. Finally, the staining was developed with 3,3'-diaminobenzidine tetrahydrochloride (DAB, Dako, Glostrup, Denmark).

\section{Immunohistochemical analysis}

NDRG2 staining was evaluated separately under double-blinded conditions by two pathologists without prior knowledge of the clinical status of the specimens. NDRG2 expression was determined by the immunohistochemistry (IHC) score, which is a semi-quantitative method using the percentage and intensity of positively stained cells as we previously described [21, 22]. Positive staining was scored as follows: "0" (less than $5 \%$ positively stained cells), " 1 " (5-24\% positively stained cells), "2" (25-49\% positively stained cells), "3" (50-74\% positively stained cells), and " 4 " (75-100\% positively stained cells). The intensity was scored as follows: " 0 " (negative staining); "1" (weak staining); "2" (moderate staining); and 
"3" (strong staining). The final IHC score was generated by multiplying the percentage score by the staining intensity score. The cut-off value of NDRG2 expression was identified as the median IHC score.

\section{Follow-up}

The patients were monitored through subsequent visits every 3 months for the first 2 years and then semiannually for 5 years after primary tumor resection. Clinical examination, CEA and carbohydrate antigen 19-9 (CA19-9) detection, and chest radiography were performed every 3 months. Chest/abdominal/pelvic CT and colonoscopy were conducted every year. OS was defined as the interval from the date of primary tumor resection to the date of death from any cause or to the last follow-up. Recurrence-free survival (RFS) was defined as the interval from liver resection to the date of disease recurrence, death or the last follow-up. Random censoring was applied to patients without recurrence or death at the last follow-up date. The final follow-up visit occurred in January 2017.

\section{Statistical analysis}

Statistical analyses were performed using IBM SPSS statistics software, version 21.0 (IBM Corp., Armonk, NY, USA). The corresponding figures were drawn using GraphPad Prism v6.0 software (GraphPad Software Inc., La Jolla, CA, USA). Categorical variables are presented as percentages, and their comparisons were assessed using the Chi-square test or Fisher's exact test. Continuous variables are presented as the median (range). The Mann-Whiney $\mathrm{U}$ test was used for two-group comparisons, and the Kruskal-Wallis $\mathrm{H}$ test was used for multiple group comparisons. Survival outcomes were evaluated by the Kaplan-Meier method and compared using the log-rank test. Parameters for which $\mathrm{P}<0.05$ for OS in the univariate Cox models were further assessed in multivariate Cox models. Hazard ratios (HRs) and 95\% confidence intervals (CIs) were subsequently generated. The statistical tests used above were twosided, and a P value $<0.05$ was considered significant.

\section{Results}

\section{Patient characteristics}

The clinical information of 316 eligible patients is summarized in Table 1 . The median age at diagnosis was 59 years (range, 23-91 years) with 196 (62.0\%) males and $120(38.0 \%)$ females.

In total, $102(32.2 \%)$ patients had stage II disease, $60(19.0 \%)$ had stage III disease, and 154 (48.7\%) had stage IV disease. Among the patients with stage IV disease, $80.5 \%(124 / 154)$ had liver metastasis, and $51.6 \%(64 / 124)$ patients received curative liver resection. Regarding postoperative treatment, 220 $(69.6 \%)$ patients received adjuvant chemotherapy.

Table 1. Clinical characteristics of 316 colorectal cancer patients

\begin{tabular}{|c|c|}
\hline Parameters & Total patients $(\mathrm{n}, \%)$ \\
\hline \multicolumn{2}{|l|}{ Patient characteristics } \\
\hline Median age (years) & $59(23-91)$ \\
\hline \multicolumn{2}{|l|}{ Age, years } \\
\hline$\leq 60$ & $166(52.5)$ \\
\hline$>60$ & $150(47.5)$ \\
\hline \multicolumn{2}{|l|}{ Sex } \\
\hline Male & 196(62.0) \\
\hline Female & $120(38.0)$ \\
\hline \multicolumn{2}{|l|}{ Primary tumor location } \\
\hline Right side of the colon & $77(24.4)$ \\
\hline Left side of the colon & 133(42.1) \\
\hline Rectum & 106(33.5) \\
\hline \multicolumn{2}{|l|}{ Primary tumor differentiation } \\
\hline Well/Moderate & $262(82.9)$ \\
\hline Poor & $54(17.1)$ \\
\hline \multicolumn{2}{|l|}{ Primary tumor diameter $(\mathrm{cm})$} \\
\hline Median (range) & $4.5(1.0-21.0)$ \\
\hline$\leq 5$ & $216(68.4)$ \\
\hline$>5$ & $100(31.6)$ \\
\hline \multicolumn{2}{|l|}{ Number of harvested lymph nodes } \\
\hline Median (range) & $14(0-55)$ \\
\hline$<12$ & $129(40.8)$ \\
\hline$\geq 12$ & $187(59.2)$ \\
\hline Number of metastatic lymph nodes (median, range) & $1(0-19)$ \\
\hline \multicolumn{2}{|c|}{ T stage } \\
\hline 1 & $8(2.5)$ \\
\hline 2 & $54(17.1)$ \\
\hline 3 & $93(29.4)$ \\
\hline 4 & $161(50.9)$ \\
\hline \multicolumn{2}{|l|}{ N stage } \\
\hline 0 & $146(46.2)$ \\
\hline 1 & $119(37.7)$ \\
\hline 2 & $51(16.1)$ \\
\hline \multicolumn{2}{|l|}{ TNM stage } \\
\hline II & $102(32.2)$ \\
\hline III & $60(19.0)$ \\
\hline IV & $154(48.7)$ \\
\hline \multicolumn{2}{|l|}{ Preoperative CEA (ng/ml) } \\
\hline$\leq 5$ & $141(44.6)$ \\
\hline$>5$ & $175(55.4)$ \\
\hline \multicolumn{2}{|l|}{ Preoperative CA19-9 (U/ml) } \\
\hline$\leq 35$ & $227(71.8)$ \\
\hline$>35$ & $89(28.2)$ \\
\hline \multicolumn{2}{|l|}{ Adjuvant chemotherapy } \\
\hline Yes & $220(69.6)$ \\
\hline No & $96(30.4)$ \\
\hline
\end{tabular}

Abbreviations: CEA: carcinoembryonic antigen, CA19-9: carbohydrate antigen 19-9

\section{NDRG2 expression in CRC tumor and normal tissues}

As shown in Figure 1, NDRG2 was located mainly in the cell cytoplasm. Positive NDRG2 expression was observed in $94.6 \%(299 / 316)$ of CRC tissues. Among the 249 patients with paired normal and tumor tissues, the median IHC score for NDRG2 expression was significantly lower in tumor tissues than in tumor-adjacent normal tissues [4.50 (range $0.00-12.00$ ) vs. 10.00 (range $0.00-12.00$ ), $\mathrm{P}<0.001$; Figure 2A]. The median IHC scores for NDRG2 
expression in right-sided colon cancer, left-sided colon cancer, and rectal cancer were 4.00 (range 0.0012.00 ), 4.67 (range $0.00-12.00$ ), and 4.83 (range 0.00 12.00), respectively, and were not significantly different $(P=0.517$, Figure 2B). Similarly, the median IHC score for NDRG2 was not significantly different between metastatic CRC and non-metastatic CRC [4.00 (range $0.00-12.00$ ) vs. 5.00 (range $0.00-12.00$ ), $\mathrm{P}=$ 0.247; Figure 2C] or between primary tumor and paired liver metastases [6.00 (range 2.67-9.00) vs. 4.00 (range 2.67-9.00), $\mathrm{P}=0.700$; Figure 2D].

\section{Association of NDRG2 expression with clinicopathologic characteristics}

As shown in Figure 3, the cut-off value for NDRG2 expression was determined as a median IHC score of 4.5. Accordingly, low NDRG2 expression in tumor cells was noted in samples from $156(49.4 \%)$ patients. Subsequently, we evaluated the association of NDRG2 expression in tumor tissues with the following clinicopathologic parameters: age, sex, tumor location, tumor size, primary tumor differentiation, TNM stage, preoperative CEA and CA199 levels, and adjuvant chemotherapy. The correlations between the clinicopathologic characteristics and NDRG2 expression are shown in Table 2. NDRG2 expression was positively associated with only age $(\mathrm{P}$ $=0.044)$, and it was not correlated with the other clinicopathologic characteristics of the patients.

\section{Association between NDRG2 expression and long-term survival in CRC patients}

All patients were followed up after their primary tumor resection for a median of 42.8 months (range: 1.0-122.6 months). Overall, 119 (37.7\%) patients died of tumor progression. In the present study, the 3-year OS rate for all patients was $68.1 \%$. Among 64 patients with liver metastasis receiving liver resection, 44
(68.8\%) patients experienced tumor recurrence, and 31 $(48.4 \%)$ patients died of tumor progression.

A log-rank test and Kaplan-Meier analysis were used to calculate the effect of NDRG2 on survival. The log-rank test showed that low NDRG2 expression was related to a poorer 3-year OS rate than high NDRG2 expression $(59.9 \%$ vs. $76.6 \%, \mathrm{P}=0.017$, Figure $4 \mathrm{~A})$. Among the stage II-III patients, the 3-year OS rate was comparable between the NDRG2 low and high expression groups $(85.1 \%$ vs. $89.6 \%, \mathrm{P}=0.013$, Figure $4 \mathrm{~B})$. However, in stage IV patients, the 3 -year OS rate was significantly worse in the low NDRG2 expression group than in the high NDRG2 expression group ( $29.4 \%$ vs. $56.5 \%, P=0.002$, Figure 4 C). Similarly, low NDRG2 expression presented a significantly poorer 3 -year OS rate in patients with liver metastasis (32.2\% vs. $54.7 \%, \mathrm{P}=0.005$, Figure $5 \mathrm{~A}$ ) and liver resection (56.5\% vs. $71.9 \%, P=0.012$, Figure $5 B$ ). In addition, the 3-year RFS rates were also significantly lower in the low NDRG2 expression group than in the high NDRG2 expression group $(20.7 \%$ vs. $45.2 \%, P=0.005$, Figure 5C).

As shown in Table 3, the univariate analysis revealed that low NDRG2 expression (HR: 1.550; 95\% CI: 1.077-2.232; $\mathrm{P}=0.018$ ), colon cancer (HR: 1.880; 95\% CI: 1.247-2.835; P = 0.003), stage IV disease (HR: 6.043; 95\% CI: 3.937-9.277; P < 0.001), high preoperative CEA (HR: 2.653; 95\% CI: 1.774-3.967; P < 0.001) and high preoperative CA19-9 (HR: 2.984; 95\% CI: 2.071-4.298; $\mathrm{P}<0.001$ ) were significant negative predictors of the 3-year OS. Multivariate analysis showed that NDRG2 expression (HR: 1.499; 95\% CI: 1.0372.165; $\mathrm{P}=0.031$ ), TNM stage (HR: $5.447 ; 95 \% \mathrm{CI}: 3.475-$ 8.538; P < 0.001), preoperative CEA level (HR: 1.610; 95\% CI: 1.043-2.485; $\mathrm{P}=0.032$ ) and preoperative CA19-9 level (HR: 2.134; 95\% CI: 1.433-3.178; P <0.001) were identified as independent predictors of the 3-year OS.

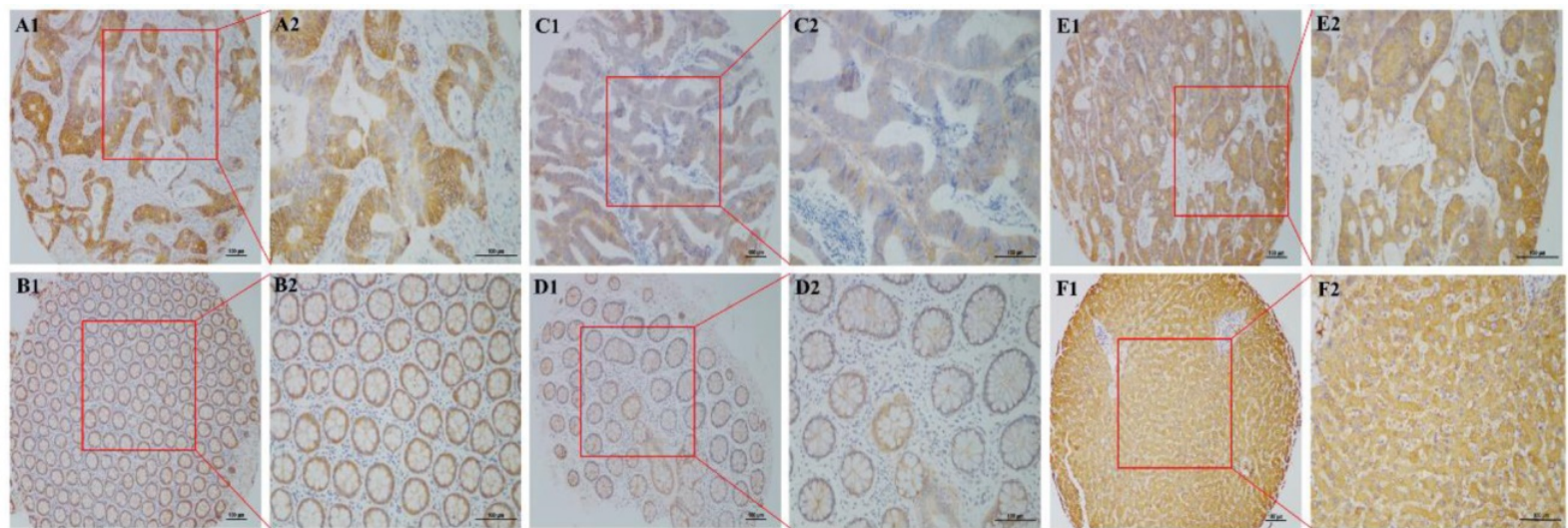

Figure 1. NDRG2 expression in primary tumor, liver metastasis and normal tissues by immunohistochemistry (IHC). (A1, A2) High NDRG2 expression in the cytoplasm of colorectal cancer (CRC) cells; (B1, B2) High NDRG2 expression in tumor-adjacent normal tissues; (C1, C2) Low NDRG2 expression in the cytoplasm of CRC cells; (D1, D2) Low NDRG2 expression in tumor-adjacent normal tissues; (E1, E2) NDRG2 expression in liver metastatic tumor tissues; (F1, F2) NDRG2 expression in normal liver tissues. Original magnification was $40 \times$ with 100- $\mu \mathrm{m}$ scale bars in A1, B1, C1, D1, E1, and F1 and 400× with 100- $\mu \mathrm{m}$ scale bars in A2, B2, C2, D2, E2, and F2. 
A

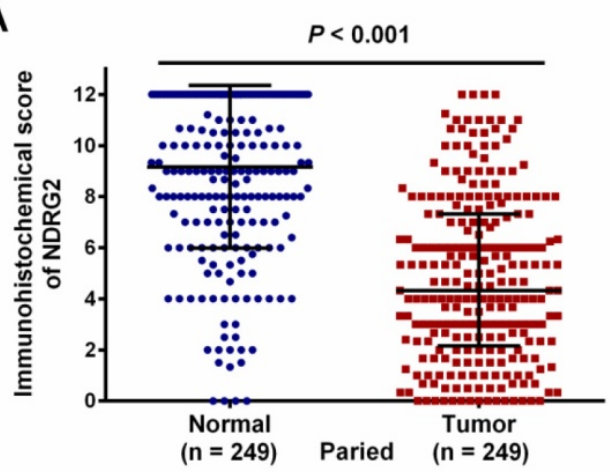

C

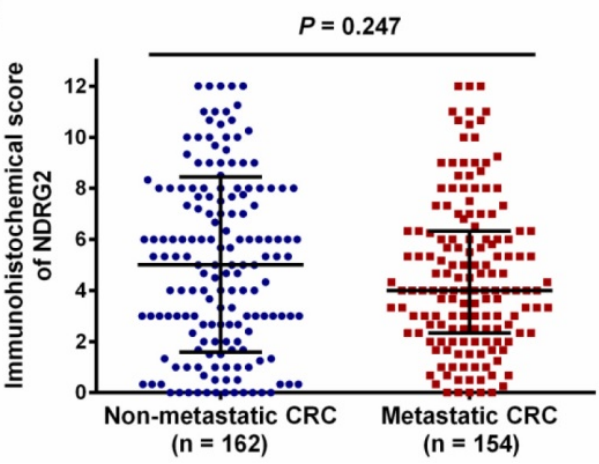

B

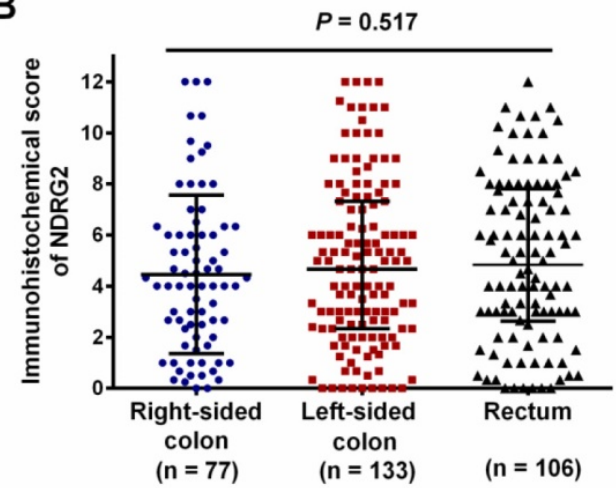

D

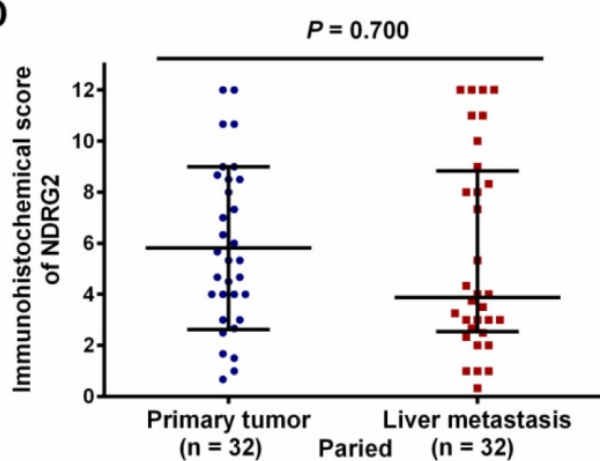

Figure 2. IHC scores for NDRG2 expression in 316 CRC patients. The red dotted line indicates the cut-off value for NDRG2 expression as the median IHC score of 4.5 .

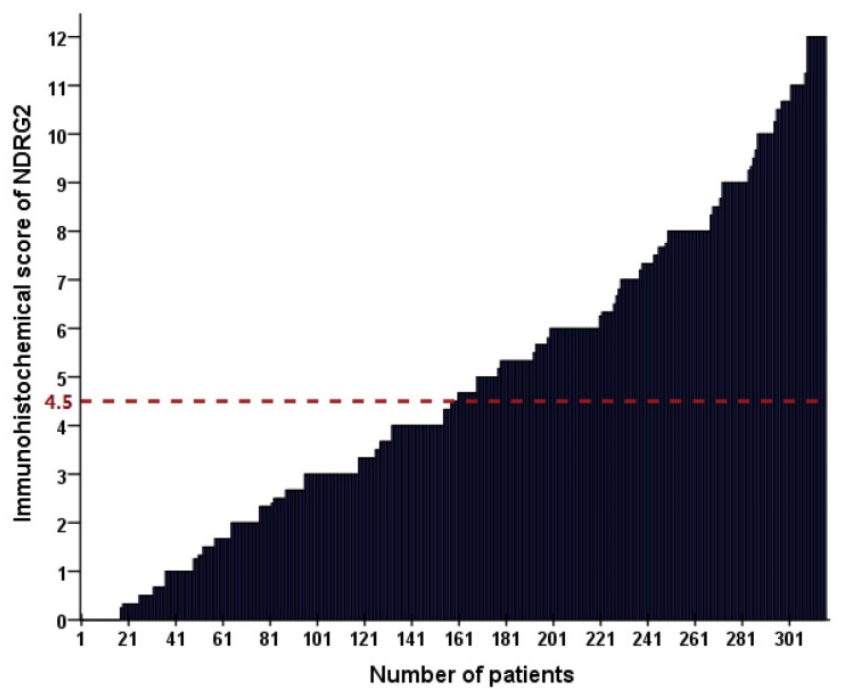

Figure 3. Comparison of NDRG2 expression levels in different subgroups of CRC patients. (A) NDRG2 expression levels were significantly lower in tumor tissue than in normal tissue $(P<0.001)$. (B) NDRG2 expression levels were comparable in patients with right-sided, left-sided and rectal tumors $(P=0.517)$. (C) NDRG2 expression levels were comparable for non-metastatic CRC and metastatic CRC $(P=0.247)$. (D) NDRG2 expression levels were comparable for primary tumor and liver metastasis $(P=0.700)$.

\section{Discussion}

Although curative surgical resection and unified adjuvant chemotherapy are commonly used in CRC patients, the definitive survival outcome of CRC remains to be elucidated. To obtain better knowledge regarding tumorigenesis and explore novel prognos- tic biomarkers for CRC, our present study evaluated NDRG2 expression in tumor tissue and normal tissues in various stage CRC patients. Our data showed that NDRG2 expression was commonly lower in human CRC tissues than in normal tissues. Furthermore, deficient NDRG2 expression in primary tumor tissue was correlated with poor OS in CRC patients, especially in those with stage IV disease. Our results also showed that NDRG2 expression was an independent prognostic factor for 3-year OS. These results implied that NDRG2 could be considered a potentially valuable prognostic indicator in CRC patients.

Several research groups have investigated and reported that decreased NDRG2 expression could contribute to reduced long-term survival in CRC patients. Chu D et al first reported that patients with reduced levels of NDRG2 mRNA had significantly shorter disease-free survival and OS than patients with normal NDRG2 mRNA expression. The results provide the first evidence that decreased NDRG2 mRNA expression in primary human CRC might be a powerful predictor of recurrence and outcome [23]. Kim YJ et al also showed that low NDRG2 expression levels were associated with shorter RFS $(\mathrm{P}=0.006)$ and a significantly unfavorable OS $(\mathrm{P}=0.001)$ [20]. However, these studies failed to evaluate the prognostic value of NDRG2 in different stages of CRC patients. Consistent with these previous results, we found that 
low NDRG2 expression was associated with a poorer 3 -year OS in all patients. Moreover, we found that the prognostic prediction effect was more significant in stage IV patients and in those with liver metastasis. In this regard, we suggest that NDRG2 is a suitable prognostic indicator in metastatic CRC patients.

Table 2. Association of NDRG2 expression and clinicopathological parameters in all patients

\begin{tabular}{|c|c|c|c|}
\hline Parameters & $\begin{array}{l}\text { Low NDRG2 } \\
\text { expression }(n=160, \%)\end{array}$ & $\begin{array}{l}\text { High NDRG2 } \\
\text { expression }(n=156, \%)\end{array}$ & P value \\
\hline \multicolumn{4}{|l|}{ Age, years } \\
\hline$\leq 60$ & $93(58.1)$ & $73(46.8)$ & 0.044 \\
\hline$>60$ & $67(41.9)$ & $83(53.2)$ & \\
\hline \multicolumn{4}{|l|}{ Sex } \\
\hline Male & $60(37.5)$ & $60(38.5)$ & 0.86 \\
\hline Female & $100(62.5)$ & $96(61.5)$ & \\
\hline \multicolumn{4}{|c|}{ Primary tumor location } \\
\hline $\begin{array}{l}\text { Right side of the } \\
\text { colon }\end{array}$ & $44(27.8)$ & $33(21.2)$ & 0.418 \\
\hline $\begin{array}{l}\text { Left side of the } \\
\text { colon }\end{array}$ & $64(40.0)$ & $69(44.2)$ & \\
\hline Rectum & $52(32.5)$ & $54(34.6)$ & \\
\hline \multicolumn{4}{|l|}{ Tumor size (cm) } \\
\hline$\leq 5$ & $104(65.0)$ & $112(71.8)$ & 0.194 \\
\hline$>5$ & $56(35.0)$ & $44(28.2)$ & \\
\hline \multicolumn{4}{|c|}{ Primary tumor differentiation } \\
\hline Well to moderate & $128(80.0)$ & $134(85.9)$ & 0.164 \\
\hline Poor & $32(20.0)$ & $22(14.1)$ & \\
\hline \multicolumn{4}{|l|}{ T stage } \\
\hline $1-3$ & $72(45.0)$ & $83(53.2)$ & 0.145 \\
\hline 4 & $88(55.0)$ & $73(46.8)$ & \\
\hline \multicolumn{4}{|l|}{ N stage } \\
\hline 0 & $68(42.5)$ & $78(50.0)$ & 0.181 \\
\hline $1-2$ & $92(57.5)$ & $78(50.0)$ & \\
\hline \multicolumn{4}{|l|}{ TNM stage } \\
\hline II-III & $75(46.9)$ & $87(55.8)$ & 0.114 \\
\hline IV & $85(53.1)$ & $69(44.2)$ & \\
\hline \multicolumn{4}{|c|}{ Preoperative CEA (ng/ml) } \\
\hline$\leq 5$ & $64(40.0)$ & $77(49.4)$ & 0.094 \\
\hline$>5$ & $96(60.0)$ & $79(50.6)$ & \\
\hline \multicolumn{4}{|c|}{ Preoperative CA199 (U/ml) } \\
\hline$\leq 35$ & $112(70.0)$ & 115(73.7) & 0.463 \\
\hline$>35$ & $48(30.0)$ & $41(26.3)$ & \\
\hline \multicolumn{4}{|c|}{ Preopeative chemotherapy } \\
\hline Yes & $34(63.0)$ & $41(78.8)$ & 0.072 \\
\hline No & $20(37.0)$ & $11(21.2)$ & \\
\hline \multicolumn{4}{|c|}{ Adjuvant chemotherapy } \\
\hline Yes & $48(30.0)$ & $48(30.8)$ & 0.882 \\
\hline No & $112(70.0)$ & $108(69.2)$ & \\
\hline
\end{tabular}

A better understanding of the molecular mechanisms by which NDRG2 functions in tumorigenesis and tumor progression will help us to elucidate prognostic results. A previous study reported that NDRG2 mRNA and protein expression was downregulated in $89 \%$ and $100 \%$ of human CRC tissue samples [24]. Our present study further showed that NDRG2 expression levels were lower in CRC tissue than in adjacent normal tissue. These results further supported the notion that NDRG2 is a tumor suppressor and is associated with progressive potential. In vitro and in vivo functional studies have shown that the cell viability, proliferation, invasion and migration of NDRG2-overexpressing cells are significantly inhibited and suppressed, which indicates that NDRG2 deficiency could contribute to cancer progression [24-26]. Accumulating evidence suggests that NDRG2 functions as a tumor suppressor by attenuating T-cell factor (TCF)/beta-catenin signaling to maintain healthy colon tissues[27, 28]. A recent study demonstrated that NDRG2 suppressed carcinogenesis by coordinately targeting glucose and glutamine transporters and multiple catalytic enzymes that are involved in glycolysis and glutaminolysis via repressing c-Myc[29]. In addition, NDRG2 loss influenced tumor-associated macrophage (TAM) polarization via the NF-kB pathway in the liver microenvironment, which promoted liver cancer metastasis [30]. This finding could partly explain why patients with low NDRG2 expression and with liver metastasis who received liver resection in our study presented with a significantly poorer 3-year OS rate. We considered that deficient NDRG2 expression might induce a higher proportion of TAM infiltration into the liver microenvironment, which may promote liver recurrence in these patients. Previous studies have demonstrated that downregulation of NDRG2 expression through inhibition of Skp2-p21/p27 axis could impede CRC differentiation and thus promoted tumor proliferation and metastasis [31]. In addition, NDRG2 is also regulated by multiple conditions, treatments, and protein/RNA entities, including hyperthermia, trichostatin A and 5-aza-2'-deoxycytidine [32]. Therefore, as a therapeutic target, upregulation of NDRG2 provides a promising strategy for the treatment of CRC.

Based on the results of our study, it may be feasible to use NDRG2 expression as a stratification parameter to categorize patients for different prognoses. In addition, the detection of NDRG2 expression could also help clinicians tailor adjuvant treatment in a comprehensive transversal approach. Therefore, we suggest that the detection of NDRG2 expression can be applied in clinical practice and serve as a supplementary diagnostic tool for CRC patients after primary tumor resection. Accordingly, if stage III-IV patients are identified as having low NDRG2 expression, the intensified or full course of adjuvant chemotherapy should be strongly recommended since the aggressive postoperative treatments might to the maximum extent reduce postoperative recurrence. Moreover, more normative follow-up measurements should be performed for patients with low NDRG2 expression tumors for timely detection of early recurrence. Otherwise, patients with increased 
NDRG2 expression tumors might benefit less from postoperative chemotherapy as they present with a favorable prognosis. Thus, enhanced postoperative chemotherapy should be avoided. Therefore, these patients can be spared the associated toxicity, cost, and inconvenience of overtreatment. In this regard, the detection of NDRG2 expression might be useful for personalizing treatment, which allows patients to maximize benefits while minimizing harm, thus providing optimal survival benefits and quality of life.

Several limitations of the present study should be acknowledged. First, the results of the current study are based mainly on a retrospective study conducted with an uncontrolled methodology and a limited number of patients. Therefore, the findings need to be validated in prospective studies to allow personalized treatment in the future. Second, the 5-year survival data were unavailable for some patients due to an insufficient follow-up duration. This limitation may have led to the underestimation of the impact of NDRG2 expression on OS in stage II-III patients. Moreover, the molecular features of tumors, such as microsatellite instability (MSI), CpG island methylator phenotype (CIMP) level, and BRAF and KRAS mutational status, were not analyzed in our study; an exploration of the relationship of NDRG2 with these molecular markers would help us further understand the impact of NDRG2 on cancer progression in CRC. Despite these limitations, our findings suggest that NDRG2 expression should be considered a routine postoperative measurement for CRC patients undergoing tumor resection.

Table 3. Univariate and multivariate Cox regression analysis of overall survival in patients with colorectal cancer after tumor resection

\begin{tabular}{|c|c|c|c|c|}
\hline \multirow[b]{2}{*}{ Variable } & \multicolumn{2}{|l|}{ Univariate } & \multicolumn{2}{|l|}{ Multivariate } \\
\hline & $\operatorname{HR}(95 \% \mathrm{CI})$ & P value & HR(95\% CI) & P value \\
\hline Age ( $\leq 60$ years vs. $>60$ years) & $1.154(0.806-1.653)$ & 0.435 & & \\
\hline Sex (male vs. female) & $0.939(0.650-1.357)$ & 0.939 & & \\
\hline Primary tumor location (colon vs. rectum) & $1.880(1.247-2.835)$ & 0.003 & $1.022(0.665-1.571)$ & 0.921 \\
\hline Tumor size (>5 cm vs. $\leq 5 \mathrm{~cm})$ & $0.700(0.465-1.055)$ & 0.088 & & \\
\hline Primary tumor differentiation (poor vs. well to moderate) & $1.549(0.996-2.408)$ & 0.052 & & \\
\hline TNM stage (IV vs. II-III) & $6.043(3.937-9.277)$ & $<0.001$ & $5.447(3.475-8.538)$ & $<0.001$ \\
\hline Preoperative CEA ( $>5 \mathrm{ng} / \mathrm{ml}$ vs. $\leq 5 \mathrm{ng} / \mathrm{ml})$ & $2.653(1.774-3.967)$ & $<0.001$ & $1.610(1.043-2.485)$ & 0.032 \\
\hline Preoperative CA199 (>35 U/ml vs. $\leq 35 \mathrm{U} / \mathrm{ml}$ ) & $2.984(2.071-4.298)$ & $<0.001$ & $2.134(1.433-3.178)$ & $<0.001$ \\
\hline Adjuvant chemotherapy (yes vs. no) & $0.838(0.570-1.232)$ & 0.369 & & \\
\hline NDRG2 expression (low vs. high) & $1.550(1.077-2.232)$ & 0.018 & $1.499(1.037-2.165)$ & 0.031 \\
\hline
\end{tabular}

A

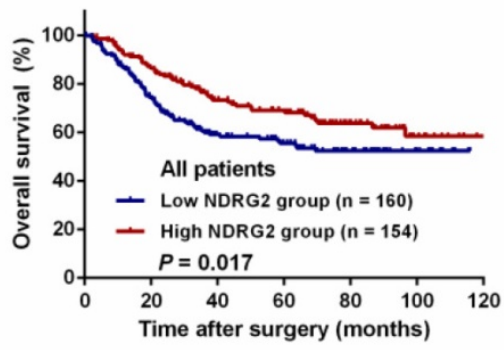

B

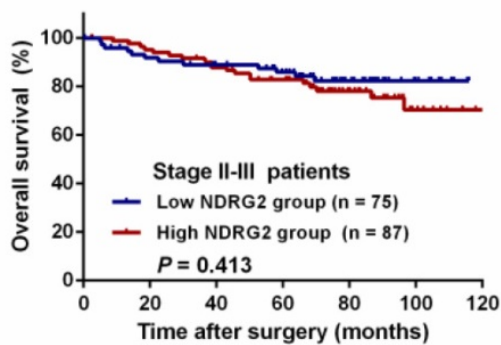

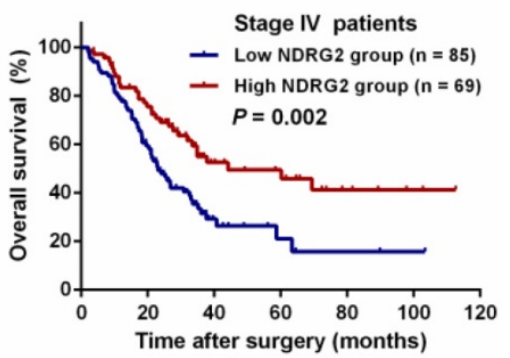

Figure 4. Kaplan-Meier overall survival (OS) curves grouped by high and low NDRG2 expression in CRC patients. (A) OS in all patients. (B) OS in patients with stage II-III disease. (C) OS in patients with stage IV disease.

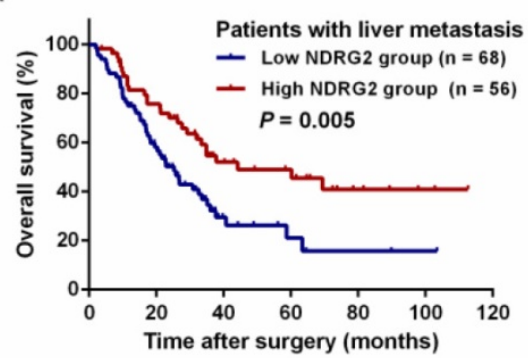

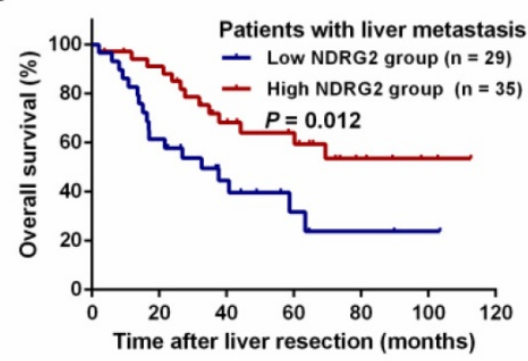

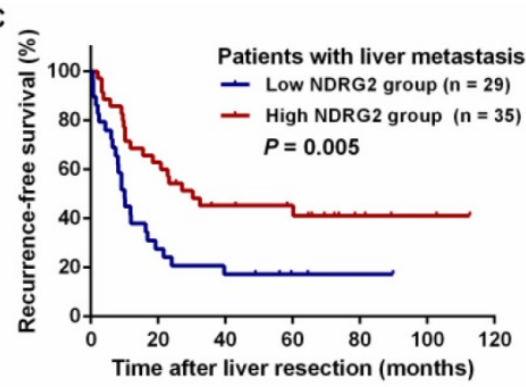

Figure 5. Kaplan-Meier curves for the comparison of long-term survival rates grouped by high and low NDRG2 expression in CRC patients with liver metastasis. (A) OS of patients with liver metastasis. (B) OS of patients with liver resection. (C) Recurrence-free survival (RFS) of patients with liver resection. 


\section{Conclusions}

The present study showed that NDRG2 expression was decreased in human CRC and that NDRG2 might be a valuable prognostic biomarker. The detection of NDRG2 expression may help oncologists evaluate the benefit of surgery and formulate individualized strategies for postoperative treatment.

\section{Acknowledgments}

We would like to thank the all colleagues of Department of Colorectal Surgery in Sun Yat-sen University Cancer Center, who have involved with performing the treatment for current study. The authenticity of this article has been validated by uploading the key raw data onto the Research Data Deposit public platform (www.researchdata.org.cn), with the approval RDD number as RDDB2019000552.

\section{Funding}

The study was supported by the Guangzhou Science and Technology Plan Projects (Health Medical Collaborative Innovation Program of Guangzhou) (grant No. 201803040019 and NO. 201400000001-4) and National Natural Science Foundation of China (grant No. 81871991 and No. 81772595).

\section{Availability of data and materials}

The datasets analysed during the current study were available from the corresponding author on reasonable request. Anyone who is interested in the information should contact fangyj@sysucc.org.cn and panzhzh@sysucc.org.cn.

\section{Competing Interests}

The authors have declared that no competing interest exists.

\section{References}

1. Chen W, Zheng R, Baade P, Zhang S, Zeng H, Bray F, et al. Cancer statistics in China, 2015. CA Cancer J Clin. 2016;66:115-32.

2. Allemani C, Matsuda T, Di Carlo V, Harewood R, Matz M, Nikšić M, et al. Global surveillance of trends in cancer survival 2000-14 (CONCORD-3): analysis of individual records for 37513025 patients diagnosed with one of 18 cancers from 322 population-based registries in 71 countries. Lancet. 2018;391:1023-75.

3. Siegel R, Miller K, Fedewa S, Ahnen D, Meester R, Barzi A, et al. Colorectal cancer statistics, 2017. CA Cancer J Clin. 2017;67:177-93.

4. Peng J, Zhang R, Zhao Y, Wu X, Chen G, Wan D, et al. Prognostic value of preoperative prognostic nutritional index and its associations with systemic inflammatory response markers in patients with stage III colon cancer. Chin J Cancer. 2017:36:96.

5. Lin J, Peng J, Zhao Y, Luo B, Zhao Y, Deng Y, et al. Early recurrence in patients undergoing curative resection of colorectal liver oligometastases: identification of its clinical characteristics, risk factors, and prognosis. J Cancer Res Clin Oncol. 2018;144:359-69.

6. Brudvik KW, Jones RP, Giuliante F, Shindoh J, Passot G, Chung MH, et al. RAS Mutation Clinical Risk Score to Predict Survival After Resection of Colorectal Liver Metastases. Ann Surg. 2019;269:120-6.

7. Gagnière J, Dupré A, Gholami S, Pezet D, Boerner T, Gönen M, et al. Is Hepatectomy Justified for BRAF Mutant Colorectal Liver Metastases?: A Multi-institutional Analysis of 1497 Patients. Ann Surg. 2018. doi: 10.1097/SLA.0000000000002968.

8. Yamashita S, Chun Y, Kopetz S, Maru D, Conrad C, Aloia T, et al. APC and PIK3CA Mutational Cooperativity Predicts Pathologic Response and Survival in Patients Undergoing Resection for Colorectal Liver Metastases. Ann Surg. 2017. doi: 10.1097/SLA.0000000000002245.

9. Melotte V, Qu X, Ongenaert M, van Criekinge W, de Bruïne A, Baldwin H, et al. The $\mathrm{N}$-myc downstream regulated gene (NDRG) family: diverse functions, multiple applications. FASEB J. 2010;24:4153-66.

10. Yao L, Zhang J, Liu X. NDRG2: a Myc-repressed gene involved in cancer and cell stress. Acta Biochim Biophys Sin (Shanghai). 2008:40:625-35.

11. Shen $\mathrm{L}, \mathrm{Qu} X, \mathrm{Li} \mathrm{H}, \mathrm{Xu} \mathrm{C}$, Wei M, Wang Q, et al. NDRG2 facilitates colorectal cancer differentiation through the regulation of Skp2-p21/p27 axis. Oncogene. 2018;37:1759-74.

12. Rong $X$, Sun $Y$, Liu D, Yin H, Peng $Y, X u$ S, et al. The pathological roles of NDRG2 in Alzheimer's disease, a study using animal models and APPwt-overexpressed cells. CNS Neurosci Ther. 2017;23:667-79.

13. Fu $\mathrm{Q}$, Gao $\mathrm{Y}$, Yang $\mathrm{F}$, Mao $\mathrm{T}$, Sun $\mathrm{Z}$, Wang $\mathrm{H}$, et al. Suppression of microRNA-454 impedes the proliferation and invasion of prostate cancer cells by promoting $\mathrm{N}$-myc downstream-regulated gene 2 and inhibiting WNT $/ \beta$-catenin signaling. Biomed Pharmacother. 2018;97:120-7.

14. Hu W, Yang Y, Fan C, Ma Z, Deng C, Li T, et al. Clinical and pathological significance of N-Myc downstream-regulated gene 2 (NDRG2) in diverse human cancers. Apoptosis. 2016;21:675-82.

15. Li S, Wang W, Li B, Chen B, Zhang B, Wang X, et al. Expression of NDRG2 in human lung cancer and its correlation with prognosis. Med Oncol. 2013;30:421.

16. Shi H, Li N, Li S, Chen C, Wang W, Xu C, et al. Expression of NDRG2 in esophageal squamous cell carcinoma. Cancer Sci. 2010;101:1292-9.

17. Yamamura A, Miura K, Karasawa H, Morishita K, Abe K, Mizuguchi Y, et al. Suppressed expression of NDRG2 correlates with poor prognosis in pancreatic cancer. Biochem Biophys Res Commun. 2013;441:102-7.

18. Gödeke J, Luxenburger E, Trippel F, Becker K, Häberle B, Müller-Höcker J, et al. Low expression of $\mathrm{N}$-myc downstream-regulated gene 2 (NDRG2) correlates with poor prognosis in hepatoblastoma. Hepatol Int. 2016;10:370-6.

19. Shen $\mathrm{L}, \mathrm{Qu} X, \mathrm{Ma} Y$, Zheng J, Chu D, Liu B, et al. Tumor suppressor NDRG2 tips the balance of oncogenic TGF- $\beta$ via EMT inhibition in colorectal cancer. Oncogenesis. 2014;3:e86.

20. Kim Y, Kang H, Yim H, Kim J, Kim J. NDRG2 positively regulates E-cadherin expression and prolongs overall survival in colon cancer patients. Oncol Rep. 2013;30:1890-8

21. Peng $\mathrm{I}, \mathrm{Ou} \mathrm{O}, \mathrm{Wu} X$, Zhang $\mathrm{R}$, Zhao $\mathrm{O}$, Jiang $\mathrm{W}$, et al. Expression of voltage-gated sodium channel Nav1.5 in non-metastatic colon cancer and its associations with estrogen receptor (ER)- $\beta$ expression and clinical outcomes. Chin J Cancer. 2017;36:89.

22. Peng J, Ou Q, Guo J, Pan Z, Zhang R, Wu X, et al. Expression of a novel CNPY2 isoform in colorectal cancer and its association with oncologic prognosis. Aging (Albany NY). 2017;9:2334-51.

23. Chu D, Zhang Z, Li Y, Wu L, Zhang J, Wang W, et al. Prediction of colorectal cancer relapse and prognosis by tissue mRNA levels of NDRG2. Mol Cancer Ther. 2011;10:47-56.

24. Hong S, Kim S, Kim E, Chang D, Kim Y. Epigenetic silencing of NDRG2 promotes colorectal cancer proliferation and invasion. J Gastroenterol Hepatol. 2016;31:164-71.

25. Golestan A, Ghaderi A, Mojtahedi Z. Effects of NDRG2 Overexpression on Metastatic Behaviors of HCT116 Colorectal Cancer Cell Line. Adv Pharm Bull. 2017;7:661-4.

26. Golestan A, Mojtahedi Z, Ghalamfarsa G, Hamidinia M, Takhshid M. The Effects of NDRG2 Overexpression on Cell Proliferation and Invasiveness of SW48 Colorectal Cancer Cell Line. Iran J Med Sci. 2015;40(5):430-9.

27. Kim J, Kim J, Kang Y, Kim K, Lee S, Choi S, et al. NDRG2 and PRA1 interact and synergistically inhibit $\mathrm{T}$-cell factor/ $\beta$-catenin signaling. FEBS Lett. 2012;586:3962-8.

28. Hwang J, Kim Y, Kang H, Jaroszewski L, Deacon A, Lee H, et al. Crystal structure of the human N-Myc downstream-regulated gene 2 protein provides insight into its role as a tumor suppressor. J Biol Chem. 2011;286:12450-60.

29. Xu X, Li J, Sun X, Guo Y, Chu D, Wei L, et al. Tumor suppressor NDRG2 inhibits glycolysis and glutaminolysis in colorectal cancer cells by repressing c-Myc expression. Oncotarget. 2015;6:26161-76.

30. Li M, Lai X, Zhao Y, Zhang Y, Li M, Li D, et al. Loss of NDRG2 in liver microenvironment inhibits cancer liver metastasis by regulating tumor associate macrophages polarization. Cell Death Dis. 2018;9:248.

31. Shen $\mathrm{L}, \mathrm{Qu}$ X, $\mathrm{Li} \mathrm{H}, \mathrm{Xu}$ C, Wei M, Wang Q, et al. NDRG2 facilitates colorectal cancer differentiation through the regulation of Skp2-p21/p27 axis. Oncogene, 2018, 37(13)

32. $\mathrm{Hu} \mathrm{W}$, Fan $\mathrm{C}$, Jiang $\mathrm{P}, \mathrm{Ma} Z$, Yan $\mathrm{X}$, Di S, et al. Emerging role of N-myc downstream-regulated gene 2 (NDRG2) in cancer. Oncotarget, 2016, 7:209-223. 